\title{
Caught in the net: Characterizing how testicular cancer patients use the internet as an information source
}

Sarah $\mathrm{Yeo}^{1}$; Bernhard Eigl ${ }^{2}$; Sherry Chan ${ }^{2}$; Christian Kollmannsberger ${ }^{2}$; Paris-Ann Ingledew ${ }^{2}$ ${ }^{1}$ University of British Columbia, Vancouver, BC, Canada; ${ }^{2} \mathrm{BC}$ Cancer Center, Vancouver, BC, Canada

Cite as: Yeo S, Eigl B, Chan S, et al. Caught in the net: Characterizing how testicular cancer patients use the internet as an information source. Can Urol Assoc J 2021 January 4; Epub ahead of print. http://dx.doi.org/10.5489/cuaj.6870

Published online January 4, 2021

$* * *$

\begin{abstract}
Introduction: Over 70\% of Canadians who use the internet search for healthcare information online. This is especially true regarding the young adult population. Testicular cancer is the most commonly diagnosed cancer in men aged 15-29. This study characterizes how testicular cancer patients access healthcare information online, and how this influences their clinical encounters and treatment decisions.

Methods: From June 2018 to January 2019, a survey consisting of 24 open and close-ended questions was distributed to testicular cancer patients at a tertiary cancer center. Survey results were evaluated using mixed methods analysis.

Results: Fifty-nine surveys were distributed, and 44 responses were received. All respondents used the internet regularly and $82 \%$ used the internet as a source of information regarding their cancer. The majority followed top hits from Google when selecting websites to view. Frequent topics searched included treatment details and survivorship concerns. Eighty-nine percent of users found online information easy to understand and $94 \%$ found it increased their understanding. For $47 \%$ of users, the internet did not influence their clinical consultation nor their treatment decision (53\%).
\end{abstract}

Conclusions: Most testicular cancer patients in this study are regular internet users and use the internet to search for testicular cancer information. Healthcare providers should recognize this and can play important roles in discussing online findings with patients to assess their background knowledge and expectations, as well as providing guidance on selecting credible online resources. The results of this study can be used to improve patient-physician communication and education. 


\section{Introduction}

Since the launch of the World Wide Web in 1989, the internet has expanded globally at a remarkable pace with current estimates showing that over $91 \%$ of Canadians have internet access. ${ }^{1}$ Health care consumers are increasingly turning to the internet as a resource, with many admitting that the Internet is the first place they look for information rather than physicians. ${ }^{2}$ This is particularly true of the young adult population who are much more likely to search for health care information online. ${ }^{3-5}$

With patients increasingly using the internet as a source of medical information, health care professionals must be equipped to help patients navigate the internet. ${ }^{6}$ Many health care training programs now define competencies related to the translation and integration of the internet into the patient encounter. As an example, CanMEDS is a physician competency framework developed by the Royal College of Physicians and Surgeons of Canada. One of the key competencies within the Communicator role states that physicians will: "Assist patients and their families to identify, access, and make use of information and communication technologies to support their care and manage their health."7 To accomplish this, practicing health care professionals should be proficient in interacting with the internet equipped patient, have knowledge of their information seeking habits and understand ways online information is used in the patient encounter.

Testicular cancer is the most commonly diagnosed cancer in men aged $15-29 .{ }^{8}$ Given the young patient demographics it is highly likely that the internet is a prevalent source of information for these patients. Little is known about how testicular cancer patients use the internet to address their unmet informational needs and to our knowledge there have been no prior publications in this area. Having this understanding would help to inform interactions for patients and their health care providers.

Our study aims to characterize how testicular cancer patients are accessing health care information online and how this influences decision making and clinical encounters. Doing so will better enable health care providers to have open discussions regarding patients' findings on the internet, translate and clarify ambiguous or false information, and direct patients to reliable websites.

\section{Methods}

From June 2018 to January 2019, testicular cancer patients at a tertiary cancer centre were invited to participate in a survey which explored their internet usage regarding their disease. Patients were included at any stage of treatment. Inclusion criteria were having a diagnosis of testicular cancer, being able to provide voluntary consent, having an adequate comprehension of the English language to understand English online resources, and age greater than 18 years.

Patients were invited to complete the survey either on paper or online. The online version was created using a local Survey Tool provided by Qualtrics and all survey data was stored in 
Canada complying with BC FIPPA regulations. Participation was anonymous and voluntary. The study was approved by the University of British Columbia Ethics Board, certificate H18-00872.

The survey consisted of 24 open and close-ended questions. A review of literature regarding patient internet use was used to develop these questions. ${ }^{6}$ The survey was developed using the best practices on survey development from the literature. ${ }^{9,10}$ This survey has been used, iteratively reviewed and refined for a process of validation in 17 other tumor sites by the research team to date. ${ }^{11-14}$ Prior to distribution, a draft survey was circulated to experts in urology and oncology education for peer-review.

Mixed methods were used to interpret the quantitative and qualitative survey results. Descriptive statistics were used to evaluate quantitative survey data. Narrative and open-ended questions were analyzed using a grounded theory approach. Themes were identified by two authors using constant comparative analysis and used to categorize survey responses. Exemplary quotes were used to provide examples.

\section{Results}

Sixty-one patients were approached regarding the study, 59 agreed to participate and 44 completed the survey for a response rate of $75 \%$. Of the 44 completed surveys, 6 were submitted online and the rest by paper. Participant demographics are shown in Table 1. All participants were male. Participants ages ranged from 23-64 years with an average age of 37 years.

\section{Internet use}

All respondents $(100 \%, n=44)$ reported using the internet, and 93\% $(n=41)$ used it regularly (between 1-4 times a day). The majority of respondents $(82 \%, n=36)$ used the internet as a source of information about their testicular cancer. We will refer to this group of participants as "users". Some reasons non-users provided for not using the internet as a source of information include being skeptical of the available information $(n=4)$, not sure where to look $(n=2)$, confused or overwhelmed $(n=2)$ and/or satisfied by the info provided by health care provider $(n=2)$.

\section{Internet search patterns}

The majority $(94 \%, n=34)$ of users used a search engine to find testicular cancer information. Of those, $100 \%(n=34)$ used Google as their principle search engine. When asked to list search terms they recalled using, 32 users provided responses. The most popular search term was "testicular cancer" $(\mathrm{n}=21)$. Other common search terms involved treatment details such as surgery specifics, chemotherapy drugs and side effects, survivorship information and epidemiology/staging (Table 2).

To select which websites to view, the majority of users followed top hits from their search engine $(81 \%, n=29)$. Others selected specific websites from reputable sources $(58 \%$, $\mathrm{n}=21)$, followed recommendations from their health care provider $(22 \%, \mathrm{n}=8)$ or 
recommendations from family and friends $(8 \%, n=3)$. Users typically reviewed 6-10 websites $(47 \%, n=17)$. Four participants viewed more than 20 websites.

\section{Websites accessed}

Twenty-four users listed names of websites they recalled visiting. Of those, the majority (58\%, $\mathrm{n}=14$ ) visited academic/institutional websites (e.g. BC Cancer, Mayo clinic, UpToDate) and websites run by non-profit organizations $(58 \%, n=14)$ (e.g. Canadian Cancer Society, American Cancer Society, Testicular Cancer Society). Commercial sites were accessed by $29 \%(n=7)$ of participants (e.g. WebMD, Wikipedia) and 13\% $(n=3)$ viewed government sites (e.g. NIH, NHS). Sixty-two percent $(n=15)$ of participants reported accessing at least one Canadian resource.

Regarding search patterns, treatment was the most commonly sought topic $(89 \%, n=32)$, followed by testicular cancer definition $(83 \%, n=30)$, incidence/prevalence $(81 \%, n=29)$, diagnosis $(78 \%, n=28)$, symptoms $(69 \%, n=25)$ and prognosis $(69 \%, n=25)$.

Ten users $(28 \%)$ accessed information about testicular cancer on social media or social networking sites. These platforms included online forums $(n=5)$, Reddit $(n=4)$, YouTube $(n=4)$, blogs $(n=3)$ and Twitter $(n=1)$.

\section{Website quality evaluation}

Forty percent $(n=14)$ of users evaluated the quality of online information all the time, while $33 \%$ $(n=12)$ evaluated some of the time, and $28 \%(n=10)$ never. Common methods included comparing with other sources $(58 \%, \mathrm{n}=21)$ and using credible sources such as academic or government websites $(50 \%, \mathrm{n}=18)$ (Figure 1$)$.

One third of users $(33 \%, n=12)$ had specific testicular cancer websites recommended to them by their health care provider. The most commonly recommended website was that of the local cancer center $(n=11)$, followed by Canadian Cancer Society $(n=4)$. All users found the recommendations to be useful. Two users reported that information they found on the internet conflicted with information provided by their health care provider. Most users $(56 \%, \mathrm{n}=20)$ did not speak with their health care provider regarding the information they found on the internet. Users stated they did not because the information online was in alignment with what was provided by their health care provider and/or they did not encounter any new information that was not already covered. Two patients reported that their health care provider specifically told them not to use the internet to search about their disease. Those that did discuss internet use with their health care provider did so to verify online information and clarify understanding of knowledge gained on the internet. 


\section{Impact of online information}

Most users $(89 \%, \mathrm{n}=32)$ found that online information available was easy to understand while $11 \%(n=4)$ found it somewhat hard to understand and none found it hard to understand. The majority $(94 \%, n=34)$ of users found the internet to be a useful source of information and that it increased their overall understanding of testicular cancer $(94 \%, n=34)$. Themes of narrative answers reported that the internet was valuable in filling knowledge gaps both pre-consult and post-consult. Pre-consult, the internet was used to prepare for the visit by getting "a summary or high-level overview", in addition to being accessible, quick and comprehensive. Post-consult the internet was used to compare and verify online information with the doctor's opinion and answer additional questions that had not been asked during the consult or there had not been time to answer. Lastly, the internet was useful in connecting with other testicular cancer patients through blogs and discussion boards.

The majority of users $(53 \%, \mathrm{n}=19)$ found that online information did not influence their treatment decisions, while $39 \%(n=14)$ found it somewhat influential and $8 \%(n=3)$ very influential. Most users $(47 \%, n=17)$ found that online information did not influence their consultation with cancer specialist, while $42 \%(n=15)$ found that it was helpful. None found that the internet made the consultation more difficult.

Users felt that the greatest strengths of the internet were ease of access $(83 \%, n=30)$, followed by volume and detail of information available $(72 \%, \mathrm{n}=26)$, discussion of differing perspectives/options $(47 \%, n=17)$ and anonymity $(44 \%, n=16)$. Topics users wish were covered more included survivorship issues such as post treatment complications, recurrence rate and coping with the trauma and anxiety. Others would have liked to have more details and personalized experiences of the treatment itself.

\section{Discussion}

All survey participants used the internet regularly and $82 \%$ searched for testicular cancer information online. These numbers are in the upper ranges when compared to previous literature in other cancer sites such as prostate cancer. ${ }^{11-15}$ The higher rates of internet usage may be explained by the younger patient demographic. ${ }^{16}$ The mean age of our survey participants was 37 years compared to 69 years in the prostate cancer study. ${ }^{15}$ It has been shown that younger generations are more likely to access the internet and online health information. ${ }^{17}$ In addition, internet use for health information has increased over time, accounting for the upward trend of when compared to older studies. ${ }^{18,19}$

It is important to explore how patients filter and select which websites to visit when looking for information online as this affects the quality of information they ultimately acquire. ${ }^{6}$ The majority of participants selected websites based on top hits returned by Google. This is similar to search strategies employed by general consumers. ${ }^{20,21}$ Unfortunately the top hits returned by Google's ranking algorithm may not necessarily be the most reliable for medical information. $^{22}$ This risk may be offset by the fact that users in this study reviewed search topics 
in greater depth (6-10 websites) than the average consumer, who typically do not view more than the top 5 links on a Google search. ${ }^{23}$

The quality of online resources can be highly variable. Previous studies have found that metrics commonly used by patients to determine reliability, such as authorship, currency and citations are lacking in many online testicular cancer resources. ${ }^{24-26}$ This highlights a potential role for health care providers to discuss with patients about how/where they obtain their online information and help them interpret what they find. ${ }^{27}$ Although the majority of participants used the internet to search for testicular cancer information, less than half spoke with their provider regarding their findings. Thus, it may be up to the health care provider to initiate and encourage these conversations. A simple question such as "What resources have you used to learn about your cancer?" may open these discussions.

The most searched topic by participants was treatment, which is congruent with prior studies in alternate cancer sites. ${ }^{11-14}$ Topics pertaining to survivorship were also heavily searched, such as prognosis, survival rate and recurrence rate. In our recent analysis of 100 testicular cancer websites, we found that prognosis was one of the least accurate topics with over half of the websites labeled incomplete. ${ }^{24}$ This shows that there are areas for improvement in online content to meet patient's needs.

Over a quarter of users reported using social media as a source of testicular cancer information. Although social media has been shown to change the methods of information dissemination and end user behaviours, there are few comprehensive studies regarding the quality of cancer information on social media platforms. ${ }^{28,29}$ This is an important area for future research as most social media platforms are tailored to express views of the lay individual and often lack metrics traditionally used to judge credibility.

The majority of respondents felt that the internet was a useful resource and increased their understanding. One noteworthy quote was: "Looking up information empowered me with knowledge and offered me reassurance. The unknown can be scary, scarier than even the worse of diagnoses or outcomes." Most users stated that online information was easy to understand. This is surprising given recent research which found that the average readability level of testicular cancer resources was challenging at a Flesh Kincaid grade level of $11 .{ }^{24}$ However this may be explained again by our younger patient demographic, as it has been shown that young adults are the most proficient in technology rich environments and that health literacy skills decline with age. ${ }^{30,31}$

Despite the benefits of the internet, about half of the respondents reported that online information did not influence their consultations with health care providers or treatment decisions. This is congruent with prior studies, which found that although the internet was a common information source for patients, physicians were considered much more trustworthy. ${ }^{32}$ As aptly stated by one patient: "They know me better than a website". 
There are several limitations to this study. First, our sample size was small, and the survey was conducted with English-speaking patients at a single geographic location in Canada thus these findings may not be generalizable to all testicular cancer patient groups. All voluntary surveys have selection bias as those who do not use the internet may be less inclined to participate. Lastly, there may be recall bias as patients who were diagnosed many years ago may not accurately remember their internet usage, and some may not be comfortable disclosing their internet habits.

\section{Conclusions}

To our knowledge this is the first study to date exploring the internet usage of testicular cancer patients and its effects on clinical interactions. We show that many patients are regular internet users and use the internet to search up information about their disease, particularity regarding treatment details and survivorship concerns. Health care providers, including urologists, oncologist and family physicians can play an important role in offering guidance on selecting credible resources, as well as discussing online findings with patients to assess their background knowledge and expectations. While many testicular cancer patients found the internet to be a useful resource to increase their understanding, it was not very influential in making treatment decisions or in clinical consultations. Hopefully these results can be used to improve patienthealth care provider communication and education. 


\section{References}

1. Statistics Canada. Ottawa, Ontario: Statistics Canada; [updated 2019 Nov 8]. https://www150.statcan.gc.ca/n1/daily-quotidien/191029/dq191029a-eng.htm. Accessed May 18, 2020.

2. Hesse BW, Moser RP, Rutten LJ. Surveys of physicians and electronic health information. N Engl J Med 2010;362:859-60.

3. Abara E, Narushima M, Abara EO. Patterns of computer and Internet usage among urology patients in two rural Northern Ontario communities. Can Urol Assoc $J$ 2010;4:37-41.

4. Cotten SR, Gupta SS. Characteristics of online and offline health information seekers and factors that discriminate between them. Soc Sci Med 2004;59:1795-806.

5. Dubowicz A, Schulz PJ. Medical information on the internet: a tool for measuring consumer perception of quality aspects. Interact J Med Res 2015;4:e8.

6. Ingledew PA, Regehr G, Tekian A, et. al. Navigating the Internet in Patient Physician Collaboration (Master's thesis). 2015. UIC http://hdl.handle.net/10027/19489.

7. Royal College of Physicians and Surgeons of Canada. Ottawa, Ontario: Royal College of Physicians and Surgeons of Canada; c2020. http://www.royalcollege.ca/rcsite/canmeds/framework/canmeds-role-communicator-e. Accessed May 18, 2020.

8. Canadian Cancer Society. Toronto, Ontario: Canadian Cancer Society; c2020. https://www.cancer.ca/en/cancer-information/cancer-type/testicular/risks/?region=on. Accessed May 18, 2020.

9. Artino Jr AR, La Rochelle JS, Dezee KJ, et al. Developing questionnaires for educational research: AMEE Guide No. 87. Med Teach 2014;36:463-74.

10. Fowler F. Survey Research Methods. Thousand Oaks, CA: SAGE Publications, Inc.; 2013.

11. Chang K, Berthelet E, Grubbs E, et al. Websites, Websites Everywhere: How Thyroid Cancer Patients Use the Internet. J Cancer Educ 2019.

12. Hamilton SN, Scali EP, Yu I, et al. Sifting Through It All: Characterizing Melanoma Patients' Utilization of the Internet as an Information Source. J Cancer Educ 2015;30:580-4.

13. McLeod J, Yu I, Ingledew PA. Peering into the Deep: Characterizing the Internet Search Patterns of Patients with Gynecologic Cancers. J Cancer Educ 2017;32:85-90.

14. Nguyen SK, Ingledew PA. Tangled in the breast cancer web: an evaluation of the usage of web-based information resources by breast cancer patients. J Cancer Educ 2013;28:662-8.

15. Bender JL, Feldman-Stewart D, Tong C, et al. Health-Related Internet Use Among Men With Prostate Cancer in Canada: Cancer Registry Survey Study. J Med Internet Res 2019;21:e14241.

16. Statistics Canada. Ottawa, Ontario: Statistics Canada; [updated 2018 Jan 29]. https://www 150.statcan.gc.ca/n1/daily-quotidien/180129/dq180129a-eng.htm. Accessed May 18, 2020. 
17. Statistics Canada. Ottawa, Ontario: Statistics Canada; [updated 2020 Jul 12]. https://www150.statcan.gc.ca/t1/tbl1/en/tv.action?pid=2710001801. Accessed Jul 12, 2020.

18. Castleton K, Fong T, Wang-Gillam A, et al. A survey of Internet utilization among patients with cancer. Support Care Cancer 2011;19:1183-90.

19. Percheski C, Hargittai E. Health information-seeking in the digital age. J Am Coll Health 2011;59:379-86.

20. Eysenbach G, Kohler C. How do consumers search for and appraise health information on the world wide web? Qualitative study using focus groups, usability tests, and indepth interviews. BMJ 2002;324:573-7.

21. Metz JM, Devine P, DeNittis A, et al. A multi-institutional study of Internet utilization by radiation oncology patients. J Radiat Oncol Biol Phys 2003;56:1201-5.

22. Page L, Brin S, Motwani R, et al. The PageRank Citation Ranking: Bringing Order to the Web (Technical report). 1999. Standord InfoLab http://ilpubs.stanford.edu:8090/422/

23. Advanced Web Ranking. Craiova, Dolj: Caphyon; c2002-20. https://www.advancedwebranking.com/ctrstudy/. Accessed May 18, 2020.

24. Yeo S, Eigl B, Ingledew PA. A fountain of knowledge? The quality of online resources for testicular cancer patients. Can Urol Assoc J 2020.

25. Prasanth AS, Jayarajah U, Mohanappirian R, et al. Assessment of the quality of patientoriented information over internet on testicular cancer. BMC cancer 2018;18:491.

26. Paffenholz P, Salem J, Borgmann H, et al. Testicular Cancer on the Web-an Appropriate Source of Patient Information in Concordance with the European Association of Urology Guidelines? J Cancer Educ 2018;33:1314-22.

27. Tan SS, Goonawardene N. Internet Health Information Seeking and the Patient-Physician Relationship: A Systematic Review. J Med Internet Res 2017;19:e9.

28. Griffiths F, Cave J, Boardman F, et al. Social networks--the future for health care delivery. Soc Sci Med 2012;75:2233-41.

29. Wang X, Chen L, Shi J, et al. What makes cancer information viral on social media? Comput Human Behav 2019;93:149-56.

30. Vézina S, Bélanger A, Sabourin P, et al. Projecting the literacy skills proficiency of the working-age population in a context of high immigration and increasing education levels (working paper). 2018. Centre Urbanisation Culture Société http://espace.inrs.ca/id/eprint/7155/.

31. Kobayashi LC, Wardle J, von Wagner C. Internet use, social engagement and health literacy decline during ageing in a longitudinal cohort of older English adults. $J$ Epidemiol Community Health (1978) 2015;69:278-83.

32. Inglehart RC, Taberna M, Pickard RK, et al. HPV knowledge gaps and information seeking by oral cancer patients. Oral Oncol 2016;63:23-9. 
$C U A J$ - Original Research

Yeo et al

Searching for testicular cancer info online

\section{Figures and Tables}

Fig. 1. Methods used to evaluate testicular cancer online information.

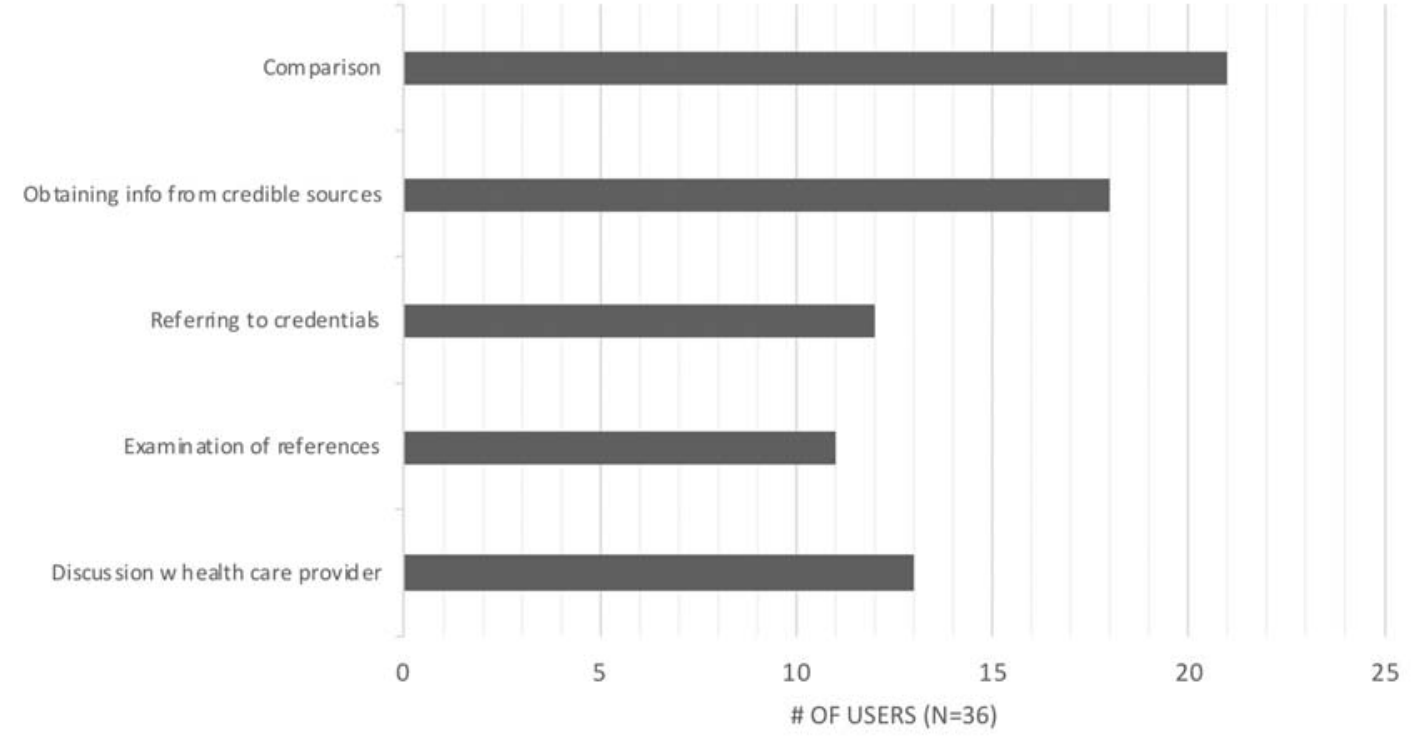

\begin{tabular}{|c|c|c|c|}
\hline & Total $(n=45)$ & Users $(n=36)$ & Non-users $(n=8)$ \\
\hline \multicolumn{4}{|l|}{ Age } \\
\hline $20-30$ years & 6 & 6 & 0 \\
\hline $30-40$ years & 25 & 19 & 6 \\
\hline $40-50$ years & 8 & 6 & 2 \\
\hline $50+$ years & 5 & 5 & 0 \\
\hline \multicolumn{4}{|l|}{ Year of diagnosis } \\
\hline $2017-2018$ & 19 & 17 & 2 \\
\hline $2014-2016$ & 12 & 11 & 1 \\
\hline $2010-2013$ & 8 & 5 & 3 \\
\hline Before 2010 & 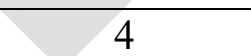 & 2 & 2 \\
\hline Unknown & 1 & 1 & 0 \\
\hline \multicolumn{4}{|l|}{ Disease stage } \\
\hline Stage 1 & 25 & 23 & 2 \\
\hline Stage 2 & 16 & 13 & 3 \\
\hline Stage 3 & 3 & 0 & 3 \\
\hline
\end{tabular}

All participants were male. 


\begin{tabular}{|c|c|c|}
\hline \multicolumn{2}{|c|}{ Search terms grouped by themes } & $\begin{array}{c}\text { Number of users who } \\
\text { used search term } \\
(\mathrm{n}=32)\end{array}$ \\
\hline \multicolumn{2}{|l|}{ Definition } & $24(75 \%)$ \\
\hline & Epidemiology & $9(28 \%)$ \\
\hline & Staging & $5(16 \%$ \\
\hline \multicolumn{2}{|l|}{ Symptoms } & $8(25 \%)$ \\
\hline \multicolumn{2}{|l|}{ Diagnosis } & $3(9 \%)$ \\
\hline \multicolumn{2}{|l|}{ Treatment } & $17(53 \%)$ \\
\hline & Surgery & $5(16 \%)$ \\
\hline & Chemotherapy & $4(13 \%)$ \\
\hline & Prosthesis & $1(3 \%)$ \\
\hline & Alternative medicine & $1(3 \%)$ \\
\hline & Side effects of treatment & $5(16 \%)$ \\
\hline \multicolumn{2}{|l|}{ Survivorship } & $11(34 \%)$ \\
\hline & Prognosis/survival rate & $10(31 \%)$ \\
\hline & Recurrence rate & $2(6 \%)$ \\
\hline Cause/prevention & 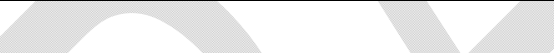 & $3(9 \%)$ \\
\hline Support groups & (n) & $2(6 \%)$ \\
\hline
\end{tabular}

